\section{Influence of drug company authorship and sponsorship on drug trial outcomes}

\author{
TONGEJI TUNGARAZA and ROB POOLE
}

\section{METHOD}

The British Journal of Psychiatry, American Journal of Psychiatry and Archives of General Psychiatry were selected as being widely read journals. They were surveyed for original data-based papers concerning psychiatric drug treatment, published between January 2000 and December 2004 inclusive. All methodologies were included (e.g. randomised controlled trials (RCTs), studies of drug levels in breast milk). Journals were searched manually and information was collected from full-text versions.

Outcomes were rated by T.T. He was aware of funding, as this was apparent in the papers. Studies were classified as reporting positive findings if they clearly stated that use of the index drug led to a better clinical outcome or was better tolerated than another treatment. Studies were classified as reporting negative findings if they clearly stated that use of a comparison treatment led to a better outcome or was better tolerated than the index drug or that there was no difference in clinical outcome or tolerability. Where the conclusions in the full text and abstract were equivocal, T.T. made a judgement as to whether the balance of findings was positive or negative.

Papers were included from all psychiatric sub-specialties. Outcome studies were included that compared an index drug with placebo, another drug or a psychological therapy. Studies were excluded if they concerned an index drug that was long established (e.g. tricyclic antidepressants, lithium, older antipsychotics) unless it was being used for a novel indication (e.g. testosterone for resistant depression). Short reports, letters to the editor, editorials, review articles and meta-analyses were excluded.

The authors' relationship with the drug company was determined from declared affiliations and conflicts of interest, or from acknowledgements. Studies were classified as industry-funded if the study was wholly or partly funded by a drug company, including funding in kind (provision of drugs and placebos, or an author who was an employee). Authors were regarded as employees if they worked full time for the company, or declared consultancy positions or shareholdings. Studies were regarded as independently funded if sufficient information was provided to exclude any of these relationships.

\section{RESULTS}

Of the 198 studies that met the inclusion criteria, 8 (4\%) lacked sufficient information on funding and were excluded. The remaining studies fell into three groups:

(a) studies funded independently of the drug industry ('independent');

(b) studies with one or more authors employed by a drug company ('industryauthored');

(c) studies funded by industry but without an employee author ('industry-sponsored').

Of these 190 studies, 33 (17\%) were published in the British Journal of Psychiatry, $98(52 \%)$ in the American Journal of Psychiatry and 59 (31\%) in the Archives of General Psychiatry. Most studies (157) concerned adults; the remainder concerned elderly people, children, or mothers and babies. Of the 132 studies that were randomised controlled trials, $112(85 \%)$ were industry-funded. In $75 \%$ of studies the index drug was an antipsychotic or an antidepressant (Table 1).

There was a significant difference between journals in reporting of negative results, the British Journal of Psychiatry being more likely to report negative findings than the other two $\left(\chi^{2}=7.99\right.$, d.f. $=2$, $P=0.0184$ ).

\section{Financial relationship with the drug industry}

Forty-four studies $(23 \%)$ were independent. Of the 146 that were industry-funded, $58(40 \%)$ also received funding from a non-industry source. Six pharmaceutical companies funded nearly half of all the studies surveyed. There were 76 industryauthored studies (40\%); of these, 64 $(84 \%)$ had authors who were employees or shareholders. Seventy studies $(37 \%)$ were industry-sponsored. 


\section{Outcomes}

Positive findings were reported in 152 $(80 \%)$ studies, whereas $38(20 \%)$ reported negative findings. Independent studies were more likely to report negative findings than industry-funded studies. Sixteen $(36 \%)$ of the 44 independent studies reported negative findings compared with $22(15 \%)$ of the industry-funded studies. The difference was statistically significant (Yates' corrected $\chi^{2}=8.3$, d.f. $\left.=1, P=0.004\right)$. Only two $(3 \%)$ of the 76 industry-authored studies reported negative findings. The difference between this group and the independent studies was highly statistically significant (Yates' corrected $\chi^{2}=22.29$, d.f. $=1$, $P<0.0001)$. A similar statistically significant difference was observed in the reporting of negative findings between industryauthored and industry-sponsored studies (Yates' corrected $\chi^{2}=17.18, \quad$ d.f. $=1$, $P<0.0001)$. There was no significant difference between independent and industrysponsored studies in reporting of positive or negative findings $\left(\chi^{2}=0.44\right.$, d.f. $=1$, $P=0.51$ )

\section{DISCUSSION}

The involvement of a drug company employee seems to exert a powerful effect on study outcome, whereas merely accepting industry sponsorship appears to have little or no effect. This finding is both novel

TONGEII TUNGARAZA, MRCPsych, ROB POOLE, FRCPsych, North East Wales Trust, UK

Correspondence: Dr Rob Poole, Pwll Glas Resource Centre, Pwll Glas Road, Mold, Flintshire CH7 IRA, UK.Tel: +44 (0) 1352 750252; email: eliphaz@doctors.org.uk

(First received 22 March 2006, final revision 7 February 2007, accepted 28 February 2007)

and counter-intuitive. One might expect that the difference between the two forms of industry funding would be subtle. In fact, the difference is highly statistically significant, in contrast to the lack of difference between studies with financial sponsorship only and fully independent studies.

There are some factors that might have confounded our findings. There were more RCTs among the industry-funded studies. Unlike other investigators, we included all methodologies because the number of independent RCTs in psychiatry is small. It might be that RCTs are intrinsically more likely to produce positive findings. Equally, they might be particularly vulnerable to being abandoned when preliminary findings are not promising (Henry et al, 2005). We did not assess the scientific quality of different studies. It is possible that independent studies tend to be statistically underpowered and that this leads to overreporting of negative findings (Djulbergovic et al, 2000; Procyshyn et al, 2004).
Our findings are unlikely to be solely due to these factors. All previous studies comparing industry-funded RCTs with independent ones have shown that the former are more likely to report positive findings. If industry-funded studies are less likely to be underpowered or methodologically flawed, then one would expect that the reporting of negative findings would be similar in the industry-authored and industry-sponsored groups, whereas actually the sponsored and independent studies were similar. We seem to have found an 'all or nothing' effect related to the involvement of a drug company employee.

In conclusion, we have confirmed previous findings that industry-funded studies are less likely to report negative findings. Our novel finding is that this effect appears to be largely or exclusively due to the presence of a company employee among the authorship. This finding requires replication with attention to differences in studies' methodological rigour and statistical power, in order to exclude these as confounding variables.

\section{REFERENCES}

Bekelman, J. E., Li, Y. \& Gross, P. C. (2003) Scope and impact of financial conflict of interest in biomedical research. A systematic review. JAMA, 289, 454-465.

Bodenheimer, T. (2000) Uneasy alliance. Clinical investigators and the pharmaceutical industry. New England Journal of Medicine, 342, 1539-1543.

Djulbergovic, B., Lacevic, M., Cantor, A., et al (2000) The uncertainty principle and industry-sponsored research. Lancet, 356, 635-638.

Henry, D. A., Kerridge, I. H., Hill, S. R., et al (2005)

Medical specialists and pharmaceutical industrysponsored research: a survey of the Australian experience. Medical Journal of Australia, 182, 557-560.

Komsaroff, P. A. \& Kerridge, I. H. (2002) Ethical issues concerning the relationship between medical practitioners and pharmaceutical industry. Medical Journal of Australia, 176, II8-121.

Lexchin, J., Bero, L. A., Djulbegovic, B., et al (2003)

Pharmaceutical industry sponsorship and research outcome and quality: systematic review. BMJ, 326 $1167-1170$

Procyshyn, R. M., Chau, A., Fortin, P., et al (2004) Prevalence and outcomes of pharmaceutical industrysponsored clinical trials involving clozapine, risperidone or olanzapine. Canadian Journal of Psychiatry, 49 $601-606$. 